\title{
Biographische Angaben zu den Beitragsautor*innen
}

Bettina Brohmann ist Soziologin und als Forschungskoordinatorin für transdisziplinäre Nachhaltigkeitswissenschaften am Öko-Institut e.V. im Büro Darmstadt tätig. Im Bereich Nukleartechnik und Anlagensicherheit arbeitet sie zu sozialwissenschaftlichen Aspekten soziotechnischer Transformationen, insbesondere in den Bereichen Kommunikation, Partizipation und Akteurskooperationen in der Energiewende sowie zum Thema Lernende Organisationen u.a. im Standortauswahlverfahren für ein Endlager hochradioaktiver Abfälle in Deutschland.

\section{E Mail: b.brohmann@oeko.de}

Achim Brunnengräber ist Politikwissenschaftler und Privatdozent am Fachbereich Politik- und Sozialwissenschaften der FU Berlin. Am Forschungszentrum für Umweltpolitik (FFU) arbeitet er zu den gesellschaftlichen Dimensionen und Dynamiken bei der Entsorgung hochradioaktiver Abfälle und zur Politischen Ökonomie der Elektromobilität. Seine Forschungsschwerpunkte sind die Energie-, Klima- und Umweltpolitik, globale politische Ökonomie, Globale Governance, sozial-ökologische Transformationsprozesse sowie NGOs und soziale Bewegungen.

E Mail: achim.brunnengraeber@fu-berlin.de

Saleem Chaudry ist Geologe und arbeitet als Wissenschaftler im Bereich Nukleartechnik und Anlagensicherheit des Öko-Instituts. Seine Schwerpunkte sind die Zwischenlagerung und Entsorgung radioaktiver Abfälle sowie Aspekte der Partizipation, insbesondere im Rahmen des Standortauswahlverfahrens für ein Endlager in Deutschland. Darüber hinaus befasst er sich mit verschiedenen umweltrelevanten geologischen Fragestellungen wie der Abscheidung und Speicherung von $\mathrm{CO}_{2}$ in geeigneten Speichergesteinen.

E Mail: s.chaudry@oeko.de 
Maria Rosaria Di Nucci ist Energieökonomin und wissenschaftliche Mitarbeiterin am Forschungszentrum für Umweltpolitik (FFU), Fachbereich Politik- und Sozialwissenschaften an der Freien Universität Berlin. Sie arbeitet als Projektleiterin der EU-geförderten Horizon 2020-Projekte WinWind (zur sozialen Akzeptanz der Windenergie) und COME RES (zu Bürgerenergie) sowie an Projekten zur Endlagerstandortsuche für hoch radioaktiver Abfälle. Ihre Arbeits- und Forschungsschwerpunkte sind vergleichende Energie- und Klimapolitik, Atompolitik im internationalen Vergleich, Förderinstrumente für erneuerbare Energien, Evaluationsmethodik und Impact Assessment, Multi-Level-Governance sowie soziale Akzeptanz von umstrittenen Technologien.

\section{E Mail: dinucci@zedat.fu-berlin.de}

Stefanie Enderle ist Diplom-Sozialwissenschaftlerin und wissenschaftliche Mitarbeiterin in der Forschungsgruppe "Endlagerung als soziotechnisches Projekt « am Institut für Technikfolgenabschätzung und Systemanalyse (ITAS) am Karlsruher Institut für Technologie (KIT). Studium der Sozialwissenschaften an der Universität KoblenzLandau, Campus Landau. Seit 2012 wissenschaftliche Mitarbeiterin am KIT u.a. am Lehrstuhl für Soziologie des Wissens und dem Methodenlabor des House of Competence mit dem Arbeitsschwerpunkt Methoden der quantitativen und qualitativen Sozialforschung. Seit 2019 wissenschaftliche Mitarbeiterin am ITAS in den Projekten »Öffentlichkeitsbeteiligung bei der Endlagersuche (ÖB-Endlager)« und »Konzepte und Maßnahmen zum Umgang mit sozio-technischen Herausforderungen bei der Entsorgung radioaktiver Abfälle (SOTEC-radio)«.

E Mail: stefanie.enderle@kit.edu

Daniel Häfner ist Kulturwissenschaftler und arbeitet am Forschungszentrum für Umweltpolitik (FFU) der FU Berlin in verschiedenen Projekten zu Fragen der Endlagerstandortsuche hoch radioaktiver Abfälle. Seine Forschungsschwerpunkte sind Soziale Bewegungen, die historische Aufarbeitung des Atomkonfliktes sowie die Begleitforschung zu Transformationsprozessen insbesondere der Lausitz.

E Mail: daniel.haefner@fu-berlin.de

Peter Hocke ist Sozialwissenschaftler und Leiter der Forschungsgruppe »Endlagerung als soziotechnisches Projekt« am Institut für Technikfolgenabschätzung und Systemanalyse (ITAS) am Karlsruher Institut für Technologie (KIT). Studium der Politischen Wissenschaft, Philosophie und Soziologie an der Universität Regensburg und der FU Berlin. Seit 2001 Projekte und Veröffentlichungen zu Fragen der nuklearen Entsorgung und Mitarbeiter am ITAS, seit 2006 Mitglied der BMU-Expertengruppe Schweizer Tiefenlager (www.escht.de). Seit Januar 2019 ist er Leiter des transdisziplinären Arbeitspakets »Handlungsfähigkeit und Flexibilität« im Forschungsverbund »Transdisziplinäre Forschung zur Entsorgung hochradioaktiver Abfälle in Deutschland« (TRANSENS, 
2019-2024) und Co-Sprecher des Forschungsverbundes. Arbeitsschwerpunkte: Technikfolgenabschätzung, Governance, Technikkonflikte und Endlagerforschung.

E Mail: hocke@kit.edu

Ana María Isidoro Losada ist Politikwissenschaftlerin und wissenschaftliche Mitarbeiterin am Forschungszentrum für Umweltpolitik (FFU), Fachbereich Politik- und Sozialwissenschaften an der Freien Universität Berlin. Ihre Arbeits- und Forschungsschwerpunkte sind soziotechnische Transformation, Energie- und Ressourcenpolitik, MultiLevel-Governance sowie politische Herrschaftsformen - mit besonderem Schwerpunkt auf politische Strukturen und Prozesse.

\section{E Mail: isidoro.losada@fu-berlin.de}

Sophie Kuppler ist Umweltwissenschaftlerin und wissenschaftliche Mitarbeiterin in der Forschungsgruppe »Endlagerung als soziotechnisches Projekt « am Institut für Technikfolgenabschätzung und Systemanalyse (ITAS) am Karlsruher Institut für Technologie (KIT). Studium der Umweltwissenschaften an der Universität Cottbus, der Yarmouk University, Jordanien und der Roskilde University, Dänemark. Seit 2009 wissenschaftliche Mitarbeiterin am ITAS in diversen Projekten zu sozialwissenschaftlichen Fragen der Endlagerung hochradioaktiver Abfälle. Promotion 2016 an der Universität Stuttgart zum Thema »Effekte deliberativer Ereignisse in der Endlagerpolitik: Deutschland und die Schweiz im Vergleich von 2001 bis 2010«. Forschungsschwerpunkte: Nukleare Entsorgung, Geothermie, Long-term Governance, Technikkonflikte.

E Mail: sophie.kuppler@kit.edu.

Erik Laes ist Postdoc-Wissenschaftler an der Technischen Universität Eindhoven in der Forschungsgruppe Philoso-phie \& Ethik, Fachbereich Industrial Engineering \& Innovation Sciences. Seine Arbeits - und Forschungsschwer-punkte sind Philosophie und Ethik von Methoden der Technikfolgenabschätzung einschließlich Szenario-Analyse, Nachhaltigkeitsbewertung, Kosten-Nutzen-Analyse, ethische Matrizen und partizipatorische Methoden. Darüber hin-aus beschäftigt er sich mit Energiethemen, insbesondere Atomenergie, Atommüllentsorgung und intelligente Netze/intelligentes Energieverhalten.

E Mail: e.j.w.laes@tue.nl

Andreas Lösch ist Soziologe und Leiter der Forschungsgruppe »Soziotechnische $\mathrm{Zu}$ künfte und Policies« am Institut für Technikfolgenabschätzung und Systemanalyse (ITAS) des Karlsruher Instituts für Technologie (KIT) sowie Privatdozent an der KITFakultät für Geistes- und Sozialwissenschaften. Seine Schwerpunkte in Forschung und Lehre liegen u.a. im Bereich der "Science and Technology Studies« (STS) und der 
Entwicklung von Theorien und Methoden des Vision Assessements in der Technikfolgenabschätzung.

E Mail: andreas.loesch@kit.edu

Melanie Mbah ist Geografin und wissenschaftliche Mitarbeiterin am Öko-Institut. Ihre Arbeits- und Forschungsschwerpunkte liegen im Bereich der Endlager-Governance, der Energiewende und der Transdisziplinarität. Hierbei untersucht sie vor allem Aspekte der Partizipation und partizipativer Formate, des Lernens in und zwischen Organisationen, der geografischen Raumforschung (insbesondere Raumwahrnehmung und Ausprägung räumlicher Identitäten) sowie der Weiterentwicklung und Anwendung transdisziplinärer Methoden und Ansätzen in soziotechnischen Zusammenhängen.

E Mail:m.mbah@oeko.de

Lutz Mez ist Politikwissenschaftler und Privatdozent der FU Berlin. Nach einer Lehre als Industriekaufmann und dem Studium der Politikwissenschaft, Soziologie, politischen Ökonomie und skandinavischen Sprachen war er in außeruniversitären Forschungseinrichtungen tätig. Am Otto-Suhr-Institut für Politikwissenschaft der FU Berlin war er seit 1984 wissenschaftlicher Mitarbeiter/Assistent. Er ist Mitbegründer des Forschungszentrums für Umweltpolitik (FFU), dessen Geschäftsführer er bis April 2010 war. Von 2009-2017 war er zudem Koordinator des Interdisziplinären Zentrums »Berlin Centre for Caspian Region Studies« (BC CARE) der FU Berlin. Sein Hauptforschungsinteresse gilt der Energie- und Umweltpolitik von Industrieländern unter besonderer Berücksichtigung der Atom-, Klimaschutz-, Gas- und Elektrizitätspolitik. Außerdem beschäftigt er sich mit Wissenstransfer und Capacity Building in Energie- und Klimapolitikprozessen insbesondere in Mittel- und Osteuropa und den Nachfolgestaaten der Sowjetunion.

E Mail: lutz.mez@fu-berlin.de

Roman Seidl ist Industriekaufmann und Diplompsychologe. Im Bereich Endlagerung radioaktiver Abfälle war er mehrere Jahre am Transdisziplinaritätslabor der ETH Zürich tätig. Darüber hinaus beschäftigte er sich mit soziotechnischen Systemen und dem gesellschaftlichen Bezug zu Umweltrisiken, an den Schnittstellen zwischen den Disziplinen und zwischen Wissenschaft und Praxis. Aktuell ist er beschäftigt am Institut für Radioökologie und Strahlenschutz der Universität Hannover.

E Mail: seidl@irs.uni-hannover.de

Jan Sieveking ist Student der Politikwissenschaften und studentischer Mitarbeiter am Forschungszentrum für Umweltpolitik (FFU) der FU Berlin. Mit dem Thema Atommüll und Endlagerung kam er in der Vergangenheit bereits im Rahmen seines zivilgesellschaftlichen Engagements in Kontakt. Am FFU verbindet er sein persönliches Interesse 
für Umweltpolitik mit den Inhalten seines theoretisch-politikwissenschaftlichen Studiums.

E Mail: j.sieveking@fu-berlin.de

Ulrich Smeddinck ist Rechtswissenschaftler am Institut für Technikfolgenabschätzung und Systemanalyse (ITAS) am Karlsruher Institut für Technologie (KIT) und lehrt als apl. Professor an der Juristischen und Wirtschaftswissenschaftlichen Fakultät der Martin-Luther-Universität Halle-Wittenberg und am KIT. Wissenschaftliche Tätigkeit an den Universitäten Cottbus, Lüneburg, Southampton und Speyer. 2013 bis 2019 stellvertretender Leiter des Institutes für Rechtswissenschaften an der TU Braunschweig. Seit Januar 2019 ist er Leiter des transdisziplinären Arbeitspakets "Dialoge und Prozessgestaltung in Wechselwirkung von Recht, Gerechtigkeit und Governance« im Forschungsverbund »Transdisziplinäre Forschung zur Entsorgung hochradioaktiver Abfälle in Deutschland« (TRANSENS, 2019-2024). Forschungsschwerpunkte: rechtswissenschaftliche und transdisziplinäre Forschung zur nuklearen Entsorgung, Umweltrecht und Governance.

E Mail: ulrich.smeddinck@kit.edu

Oliver Sträter ist Professor für Arbeit und Organisationspsychologie an der Universität Kassel nachdem er zuvor in der Kerntechnik und der europäischen Flugsicherheit tätig war. Er forscht und lehrt zu allen Aspekten menschlicher Zuverlässigkeit auf unterschiedlichsten Abstraktionsebenen eines Systems von individuellen Fehlern bis hin $\mathrm{zu}$ gesellschaftlichen Problematiken. Grundverständnis seiner Arbeit ist, dass für eine erfolgreiche und sichere Gestaltung von Systemen die Eigenschaften menschlicher Informationsverarbeitung zu berücksichtigen sind und Sicherheitsmängel immer auf mangelndes Design von Systemen hinsichtlich menschlicher Eigenschaften beruht.

E Mail: straeter@uni-kassel.de. 


\section{Politikwissenschaft}

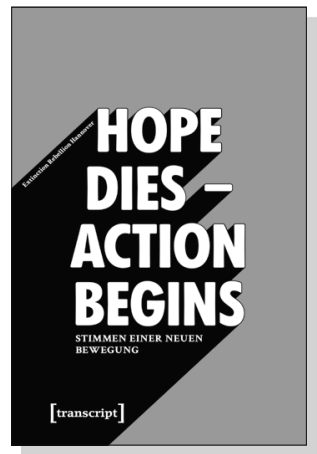

Extinction Rebellion Hannover

"Hope dies - Action begins":

Stimmen einer neuen Bewegung

2019, 96 S., kart.

7,99€ (DE), 978-3-8376-5070-9

E-Book: kostenlos erhältlich als Open-Access-Publikation, ISBN 978-3-8394-5070-3

EPUB: kostenlos erhältlich als Open-Access-Publikation, ISBN 978-3-7328-5070-9

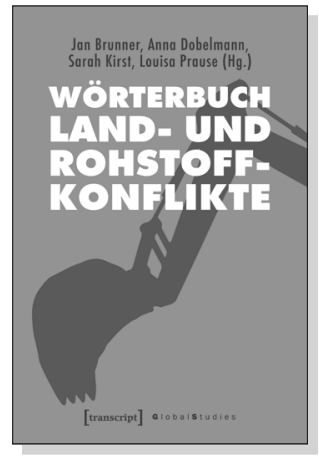

Jan Brunner, Anna Dobelmann,

Sarah Kirst, Louisa Prause (Hg.)

\section{Wörterbuch Land- und Rohstoffkonflikte}

2019, 326 S., kart., Dispersionsbindung, 1 SW-Abbildung 24,99€ (DE), 978-3-8376-4433-3

E-Book: 21,99 € (DE), ISBN 978-3-8394-4433-7

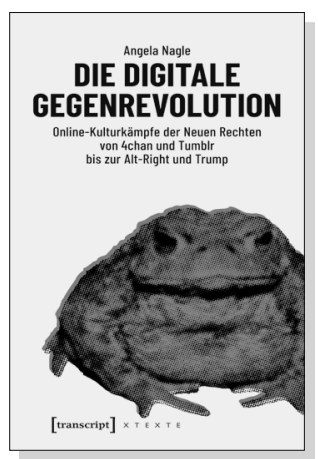

Angela Nagle

\section{Die digitale Gegenrevolution}

Online-Kulturkämpfe der Neuen Rechten

von 4chan und Tumblr bis zur Alt-Right und Trump

2018, 148 S., kart.

$19,99 €(D E), 978-3-8376-4397-8$

E-Book: 17,99 € (DE), ISBN 978-3-8394-4397-2

EPUB: $17,99 €$ (DE), ISBN 978-3-7328-4397-8 


\section{Politikwissenschaft}

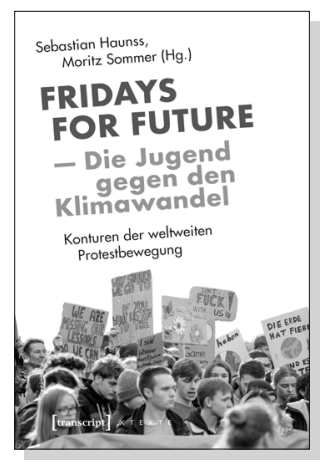

Sebastian Haunss, Moritz Sommer (Hg.)

Fridays for Future -

Die Jugend gegen den Klimawandel

Konturen der weltweiten Protestbewegung

Oktober 2020, 264 S., kart.

$22,00 €(D E), 978-3-8376-5347-2$

E-Book: kostenlos erhältlich als Open-Access-Publikation

PDF: ISBN 978-3-8394-5347-6

ISBN 978-3-7328-5347-2

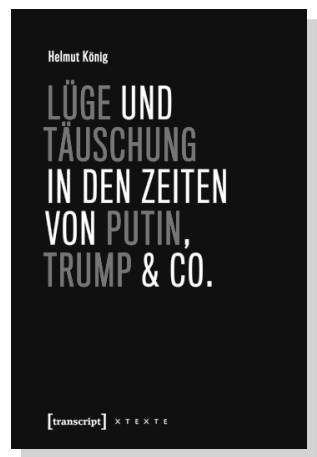

Helmut König

Lüge und Täuschung

in den Zeiten von Putin, Trump \& Co.

September 2020, 360 S., kart.

$29,50 €(D E), 978-3-8376-5515-5$

E-Book:

PDF: $26,99 €$ (DE), ISBN 978-3-8394-5515-9

EPUB: $26,99 €$ (DE), ISBN 978-3-7328-5515-5

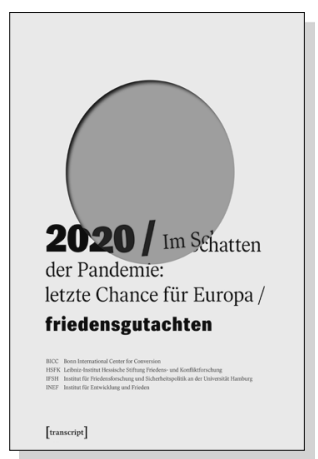

BICC Bonn International Center for Conversion, HSFK Leibniz-Institut Hessische Stiftung Friedens- und Konfliktforschung, IFSH Institut für Friedensforschung und Sicherheitspolitik an der Universität Hamburg, INEF Institut für Entwicklung und Frieden

\section{Friedensgutachten 2020}

Im Schatten der Pandemie: letzte Chance für Europa

Juni 2020, 160 S., kart., 33 Farbabbildungen

15,00€ (DE), 978-3-8376-5381-6

E-Book: kostenlos erhältlich als Open-Access-Publikation PDF: ISBN 978-3-8394-5381-0 
